\title{
Sensitization of Candida albicans to terbinafine by berberine and berberrubine
}

\author{
PIKLING LAM ${ }^{1}$, STANTON HON LUNG KOK ${ }^{2}$, KENNETH KA HO LEE ${ }^{2}$, KIM HUNG LAM ${ }^{1}$, \\ DESMOND KWOK PO HAU ${ }^{3}$, WAI YEUNG WONG ${ }^{4}$, ZHAOXIANG BIAN $^{3}$, \\ ROBERTO GAMBARI $^{5}$ and CHUNG HIN CHUI ${ }^{1,3}$
}

\author{
${ }^{1}$ State Key Laboratory of Chirosciences, Department of Applied Biology and Chemical Technology, \\ The Hong Kong Polytechnic University; ${ }^{2}$ School of Biomedical Sciences, Chinese University of Hong Kong; \\ ${ }^{3}$ Clinical Division, School of Chinese Medicine, ${ }^{4}$ Department of Chemistry, Hong Kong Baptist University, Hong Kong, SAR, \\ P.R. China; ${ }^{5}$ Department of Life Sciences and Biotechnology, Section of Biochemistry and Molecular Biology, \\ University of Ferrara, Ferrara, Italy
}

Received September 22, 2015; Accepted November 27, 2015

DOI: $10.3892 /$ br.2016.608

\begin{abstract}
Candida albicans (C. albicans) is an opportunistic fungal pathogen, particularly observed in immunocompromised patients. C. albicans accounts for 50-70\% of cases of invasive candidiasis in the majority of clinical settings. Terbinafine, an allylamine antifungal drug, has been used to treat fungal infections previously. It has fungistatic activity against $C$. albicans. Traditional Chinese medicines can be used as complementary medicines to conventional drugs to treat a variety of ailments and diseases. Berberine is a quaternary alkaloid isolated from the traditional Chinese herb, Coptidis Rhizoma, while berberrubine is isolated from the medicinal plant Berberis vulgaris, but is also readily derived from berberine by pyrolysis. The present study demonstrates the possible complementary use of berberine and berberrubine with terbinafine against $C$. albicans. The experimental findings assume that the potential application of these alkaloids together with reduced dosage of the standard drug would enhance the resulting antifungal potency.
\end{abstract}

Correspondence to: Professor Roberto Gambari, Department of Life Sciences and Biotechnology, Section of Biochemistry and Molecular Biology, University of Ferrara, Via Fossato di Mortara 74, I-44121 Ferrara, Italy

E-mail: gam@unife.it

Dr Chung Hin Chui, Clinical Division, School of Chinese Medicine, Hong Kong Baptist University, Jockey Club School of Chinese Medicine Building, 7 Baptist University Road, Hong Kong, SAR, P.R. China

E-mail: chchui@hkbu.edu.hk

Key words: antifungal, berberine, berberrubine, C. albicans, terbinafine

\section{Introduction}

The incidence of Candida infections has increased in patients who receive immunosuppressive therapy, cancer chemotherapy and transplantation. Among the Candida species, Candida albicans (C.albicans) is responsible for 50-70\% of cases of invasive candidiasis in the majority of clinical settings $(1,2)$. C.albicans, an opportunistic fungal pathogen that normally inhabits the mucous membranes of the gastrointestinal and female genital tracts (3), is able to attack the immunocompromised patients (4). When the normal microbial barrier is disrupted, C. albicans can invade the intestinal and female genital mucosal barriers and causes candidiasis and candidemia $(5,6)$. A cross-sectional study has reported that Candida species isolation from the vaginal mucosa was more frequent in human immunodeficiency virus (HIV)-infected patients $(29.7 \%)$ when compared with uninfected women (14.5\%). C.albicans was the most prevalent pathogen isolated in HIV-infected (52.9\%) and uninfected women (85.7\%) (7). A previous study investigated 128 Candida isolates from South Africa and 126 Cameroonian Candida isolates. Of those, $C$. albicans was responsible for the highest percentage of them, with $82.8 \%$ of South African isolates and $73.0 \%$ of the Cameroonian isolates (8). In another study, of the 103 Candida species-infected oral mucosal isolates identified in HIV patients, C. albicans accounted for the majority of Candida species (77.7\%) (9). A prospective observational study also examined the occurrence of oral Candida colonization among cancer patients in China between October 2012 and March 2013. C. albicans was the most common species isolated from patients, accounting for $30.8 \%$ in patients with pulmonary cancer $(n=78), 33.7 \%$ in patients with digestive tract malignant tumor $(n=101)$, and $12.7 \%$ in patients with hematopoietic system tumor $(n=79)$. Cancer patients are a high-risk population for Candida colonization (10).

Traditional Chinese medicines (TCMs) remain a fundamental role in the treatment of various diseases due to their long history of clinical practice and reliable therapeutic efficacy. Berberine (Fig. 1A) is an isoquinoline quaternary alkaloid 
isolated from the traditional Chinese herb, Coptidis Rhizoma (also known as Huanglian), which includes several species: Coptis chinensis French, Coptis deltoidea and Coptis teetoides $(11,12)$. Berberrubine (Fig. 1B) is isolated from the medicinal plant Berberis vulgaris (13), but is also readily derived from berberine by pyrolysis (14). Kim et al (15) studied the antimicrobial activity of berberine, berberrubine, $9-O$-acylberberrubines and 9-O-alkylberberrubines. Berberrubine exhibited a relatively weaker anti-C. albicans activity [minimum inhibitory concentration $(\mathrm{MIC})>128 \mu \mathrm{g} / \mathrm{ml}$ ] than berberine $(\mathrm{MIC}=128 \mu \mathrm{g} / \mathrm{ml}$ ), while some of the acyl derivatives (decanoyl and lauroyl berberrubine chlorides) and the alkyl derivatives (heptyl, octyl, nonyl, decyl and undecyl) showed much stronger growth inhibition against $C$. albicans, with the MICs ranging from 1-4 $\mu \mathrm{g} / \mathrm{ml}$. Park et al (16) reported the antifungal activity of 13-(substituted benzyl) berberine and berberrubine derivatives. Among them, 13-(4-tertbutylbenzyl) and 13-(4-isopropyl benzyl) berberine derivatives exhibited the strongest antifungal activity against $C$. albicans $(\mathrm{MICs}=4 \mu \mathrm{g} / \mathrm{ml})$ when compared with berberine $(\mathrm{MIC}=128 \mu \mathrm{g} / \mathrm{ml})$. The 13-(4-tertbutylbenzyl) and 13-(4-isopropyl benzyl) berberrubines showed a better anti-C. albicans activity (MICs $=16 \mu \mathrm{g} / \mathrm{ml})$ when compared with berberrubine (MIC $>128 \mu \mathrm{g} / \mathrm{ml}$ ).

TCMs can be used as complementary medicines to conventional drugs for a variety of disease treatments. Our previous study reported the complementary use of corilagin, a gallotannin identified in numerous plants, including Phyllanthus urinaria, with two chemotherapeutic drugs, cisplatin and doxorubicin, in order to lower the working concentration of these two agents and to obtain an increment in the anticancer effect. The $\mathrm{IC}_{50}$ values were $\sim 3$-fold reduced for cisplatin and $\sim 4$-fold decreased for doxorubicin with the Hep3B hepatoma cells in the presence of corilagin (17). The efficacy of standard antibiotics against bacterial strains was improved by the use of plant materials (18-21). TCMs as a complementary therapy with the standard antifungal drugs against fungal pathogens have emerged as a new choice for the treatment of infectious diseases. The present study utilizes the TCMs, berberine and berberrubine, as complementary agents with the standard antifungal drug, terbinafine, against the most common Candida species, C. albicans, responsible for the increased incidence of fungal infections.

\section{Materials and methods}

Chemical analysis. Nuclear magnetic resonance (NMR) spectra were recorded on a Bruker/Varian $500 \mathrm{MHz}$ Fourier transform spectrometer (Agilent Technologies, Santa Clara, CA, USA). ${ }^{1} \mathrm{H}$ and ${ }^{13} \mathrm{C}$-NMR spectra were recorded relative to residual protiated solvent; a positive value of the chemical shift denoted a resonance downfield from tetramethylsilane (TMS, internal standard). $J$-values were in $\mathrm{Hz}$. All the chemicals were purchased from commercial suppliers and used without further purification. All the reactions were monitored by analytical thin-layer chromatography (TLC) on Merck aluminum-precoated plates of silica gel 60 F254 with detection by spraying with 5\% (w/v) dodecamolybdophosphoric acid in ethanol or 5\% (w/v) ninhydrin in ethanol and subsequent heating.

Synthesis of natural berberrubine. Natural berberrubine was synthesized according to the reported procedure (22). Berberine
A

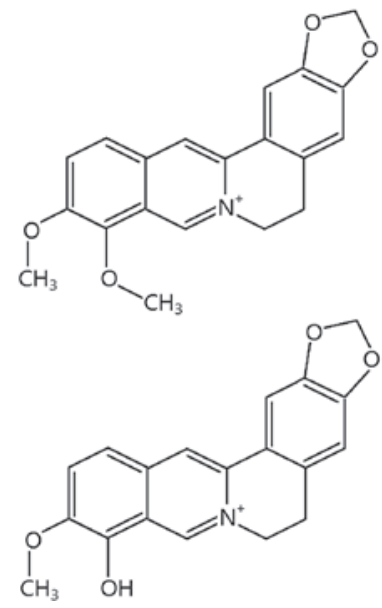

Figure 1. Chemical structure of (A) berberine and (B) berberrubine.

chloride ( $2.01 \mathrm{~g}, 5.4 \mathrm{mmol})$ was heated at $190^{\circ} \mathrm{C}$ under reduced pressure for $2 \mathrm{~h}$ and the crude product was recrystallized from chloroform and hexane to obtain the title berberrubine in $83 \%$ yield as a dark red precipitate: $\mathrm{R} f=0.29$ (dichloromethane-methanol, 15:1); ${ }^{1} \mathrm{H}-\mathrm{NMR}\left(\mathrm{CDCl}_{3}\right) \mathrm{d} 9.20(\mathrm{~s}, 1 \mathrm{H}), 7.58(\mathrm{~s}, 1 \mathrm{H})$, 7.30-7.24 (m, 2H), $6.75(\mathrm{~s}, 1 \mathrm{H}), 6.51-6.49(\mathrm{~m}, 1 \mathrm{H}), 6.06(\mathrm{~s}, 2 \mathrm{H})$, $4.42(\mathrm{~s}, 2 \mathrm{H}), 3.89$ (s, 3H), 3.09 (s, $2 \mathrm{H}) ;{ }^{13} \mathrm{C}-\mathrm{NMR}\left(\mathrm{CDCl}_{3}\right) \mathrm{d} 167.7$, $150.3,149.1,148.2,145.9,133.1,131.5,128.3,122.2,120.7,120.2$, 117.7, 108.4, 104.6, 103.3, 101.9, 56.2, 53.4, 28.6 .

Determination of MIC and minimum fungicidal concentration (MFC).C.albicans was obtained from American Type of Culture Collection (Manassas, VA, USA). The MIC values of berberine, berberrubine (kindly provided by Professor K.K.H. Lee) and terbinafine (both from Sigma-Aldrich, St. Louis, MO, USA) on $C$. albicans were determined by the broth dilution method. Briefly, different concentrations of berberine, berberrubine and terbinafine were loaded from a starting concentration of $100 \mu \mathrm{g} / \mathrm{ml}$ containing $1 \%$ dimethylsulfoxide (DMSO) as the vehicle and they were diluted serially. DMSO $(1 \%)$ was used as a vehicle control. The fungal samples were subsequently incubated at $35^{\circ} \mathrm{C}$ for $48 \mathrm{~h}$. The minimum concentrations of berberine, berberrubine and terbinafine, which induced a complete growth inhibition would be recorded as their MIC values $(16,23)$. For the determination of MFC, $10 \mu \mathrm{l}$ of the $48 \mathrm{~h}$ incubated medium was removed and plated. MFC was recorded at a concentration where no colony of fungal growth was observed. When MFC/MIC $<4$, the compound or combination would be considered as fungicidal, while when MFC/MIC $\geq 4$ the compound or combination would be considered as fungistatic. In each case, three independent experiments were conducted and each experiment was carried out in triplicates.

Sensitization test. For the sensitization investigation experiment, the (3-(4,5-dimethylthiazol-2-yl)-5-(3-carboxymethoxyphenyl)-2-(4-sulfophenyl)-2H-tetrazolium) (MTS) assay was employed (24). Briefly, C. albican cells were seeded at day 0 in the 96-well microplate. Terbinafine was added at 25, 12.5, $6.25,3.13,1.56,0.78,0.39$ and $0.2 \mu \mathrm{g} / \mathrm{ml}$, respectively, while berberine and berberrubine were added at $100 \mu \mathrm{g} / \mathrm{ml}$ together with terbinafine. After $48 \mathrm{~h}$ of incubation, MTS (Promega, Madison, WI, USA)/phenazine methosulfate (PMS) as 


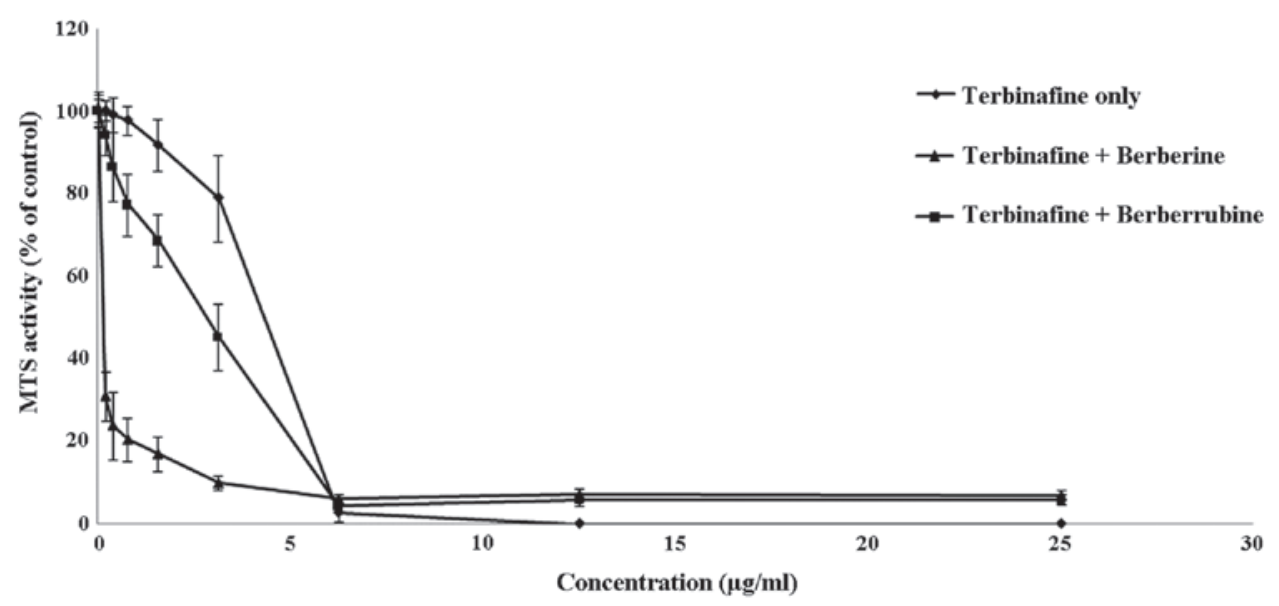

Figure 2. Sensitization of Candida albicans to terbinafine by berberine and berberrubine. Berberine and berberrubine could assist the antifungal action of the standard antifungal drug, terbinafine. The complementary activity of berebrine with terbinafine was much stronger than that of berberrubine. In each case, three independent experiments were conducted and a mean value was obtained. Each experiment was carried out in triplicates. Results are shown as mean \pm standard deviation from three independent experiments.

electron coupling agent mixed solution was added. Lastly, optical absorbance was determined at $490 \mathrm{~nm}$ using a microplate reader (Perkin Elmer Victor V) according to the manufacturer's protocol. To determine MIC and MFC values from the sensitization test, no MTS/PMS was added and the experimental procedures were conducted as mentioned before. In each case, three independent experiments were conducted and each experiment was carried out in triplicates.

Zone of inhibition study. C. albicans was used to study the effectiveness of berberine alone $(100 \mu \mathrm{g})$, berberine $(100 \mu \mathrm{g})$ plus terbinafine $(6 \mu \mathrm{g})$, berberrubine alone $(100 \mu \mathrm{g})$, and berberrubine $(100 \mu \mathrm{g})$ plus terbinafine $(6 \mu \mathrm{g})$ against its growth in culture. C. albicans was diluted with yeast mold broth and plated on the yeast mold agar plate, and the holes were created on the agar using a sterile transfer pipette. For the tested samples, $1 \%$ DMSO (as negative control) and $6 \mu \mathrm{g}$ terbinafine (as positive control) were placed in the holes of agar. The plates were subsequently incubated at $35^{\circ} \mathrm{C}$ for $48 \mathrm{~h}$ and the inhibition zones ( $\mathrm{mm}$, in terms of diameter) of fungi on the agar plates were recorded $(23,25,26)$.

\section{Results and Discussion}

Determination of MIC, MFC and sensitization assay. Terbinafine is an allylamine agent with a broad spectrum of antifungal activity. It interferes with the biosynthesis of ergosterol, an essential component of fungal cell membranes, via inhibition of the fungal enzyme squalene epoxidase. In the in vitro susceptibility tests, terbinafine have been shown to possess primarily fungicidal activity against dermatophytes, moulds and certain dimorphic fungi, but only fungistatic activity against $C$. albicans (27). The cell death mechanisms of berberine against $C$. albicans have been fully addressed in the previous studies. They involved the ability to impair mitochondrial function, generation of reactive oxygen species, targeting cell wall integrity pathway and also affecting heat shock transcription factor, HSF1 (28). The MIC and MFC values of terbinafine against $C$. albicans were determined as

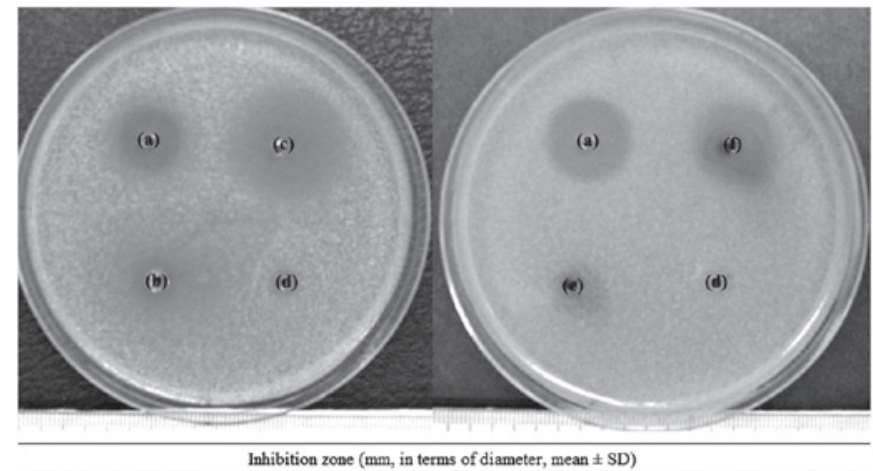

Inhibition zone ( $\mathrm{mm}$, in terms of diameter, mean $\pm \mathrm{SD})$

(a) Terbinafine (b) Berberine (c) Berberinetterbinafine (d) DMSO (e) Berberrubine (f) Berberrubinetterbinafine \begin{tabular}{rrrrrr}
$14.67 \pm 3.06$ & $6.83 \pm 1.26$ & $25.67 \pm 2.52$ & Nil & Nil & $15.83 \pm 2.25$ \\
\hline
\end{tabular}

Figure 3. Inhibition zone ( $\mathrm{mm}$, in terms of mean diameter \pm standard deviation).

6 and $24 \mu \mathrm{g} / \mathrm{ml}$, respectively. As MFC/MIC was equal to 4 , it was considered to be fungistatic. For berberine and berberrubine, their MIC values were $>100 \mu \mathrm{g} / \mathrm{ml}$. No MFC value was determined. These findings were consistent with the results reported previously (16). However, berberine and berberrubine at $100 \mu \mathrm{g} / \mathrm{ml}$ could not improve the MFC, and the MFC of terbinafine could significantly potentiate the antifungal activity of terbinafine on C. albicans as determined by the MTS/PMS assay. Berberine and berberrubine at $100 \mu \mathrm{g} / \mathrm{ml}$ could effectively assist the antifungal potential of terbinafine (Fig. 2). Notably, the complementary activity of berebrine with terbinafine was much stronger than that of berberrubine when terbinafine was loaded from $3.13,1.56,0.78,0.39$ and $0.2 \mu \mathrm{g} / \mathrm{ml}$. Therefore, berberine was more effective in amplifying the antifungal action of terbinafine when compared with its analogue, berberrubine.

Zone of inhibition study. Previous studies have reported that berberine in combination with fluconazole or miconazole showed a synergistic antifungal activity against $C$. albicans with larger inhibition zones in the agar diffusion tests (29-31). In order to evaluate the sensitivity of $C$. albicans to the combination of terbinafine and berberine or berberrubine, agar diffusion assays were conducted to determine their inhibition zones on the agar 
plates. Terbinafine alone at $6 \mu \mathrm{g}$ possessed certain fungistatic effect against $C$. albicans (inhibition zone, $14.67 \pm 3.06 \mathrm{~mm}$ ), whereas berberine or berberrubine alone showed small or no level of growth inhibition of the fungi (Fig. 3). The combination of $100 \mu \mathrm{g}$ berberine and $6 \mu \mathrm{g}$ terbinafine showed an increased inhibition zones in size (inhibition zone, $25.67 \pm 2.52 \mathrm{~mm}$ ) when compared with terbinafine alone. The result may suggest that a synergistic fungistatic activity against $C$. albicans may occur between the combined drugs even if the MIC and MFC of this combination were not improved. However, the combination of $100 \mu \mathrm{g}$ berberrubine and $6 \mu \mathrm{g}$ terbinafine did not enhance the fungal sensitivity, with no significant enlargement in size of inhibition zone. No growth inhibition of $C$. albicans could be found in $1 \%$ DMSO (vehicle control).

In conclusion, the present study reports the complementary application of berberine and berberrubine with terbinafine against the opportunistic fungal pathogen, $C$. albicans. A recent study has demonstrated that berebrine can assist fluconazole to kill fluconazole-resistant $C$. albicans (32). The present results further indicate the possible sensitization of various pathogenic fungi to other standard drugs by berberine and berberrubine, with the purpose of obtaining an increment in the antifungal potency.

\section{Acknowledgements}

The present study was supported by the Innovation Technology Commission to ABCT and HKPU [with the grant codes FRG/14-15/021, 30-14-121 and 38-40-116 (Dr C.H.C.)], as well as grant no. 03-16-176 (Professor Z.X.B.). Professor R.G. received a grant from AIRC (contract no. IG 13575).

\section{References}

1. Sanguinetti M, Posteraro B and Lass-Flörl C: Antifungal drug resistance among Candida species: Mechanisms and clinical impact. Mycoses 58 (Suppl 2): 2-13, 2015.

2. Arendrup MC: Epidemiology of invasive candidiasis. Curr Opin Crit Care 16: 445-452, 2010.

3. Richards MJ, Edwards JR, Culver DH and Gaynes RP; National Nosocomial Infections Surveillance System: Nosocomial infections in coronary care units in the United States. Am J Cardiol 82: 789-793, 1998.

4. Pu Y, Liu A, Zheng Y and Ye B: In vitro damage of Candida albicans biofilms by chitosan. Exp Ther Med 8: 929-934, 2014.

5. Schulze J and Sonnenborn U: Yeasts in the gut: From commensals to infectious agents. Dtsch Arztebl Int 106: 837-842, 2009.

6. Nenoff P, Krüger C, Schaller J, Ginter-Hanselmayer G, Schulte-Beerbühl R and Tietz HJ: Mycology - an update part 2: dermatomycoses: clinical picture and diagnostics. J Dtsch Dermatol Ges 12: 749-777, 2014.

7. Oliveira PM, Mascarenhas RE, Lacroix C, Ferrer SR, Oliveira RP, Cravo EA, Alves AP and Grassi MF: Candida species isolated from the vaginal mucosa of HIV-infected women in Salvador, Bahia, Brazil. Braz J Infect Dis 15: 239-244, 2011.

8. Dos Santos Abrantes PM, McArthur CP and Africa CW: Multi-drug resistant oral Candida species isolated from HIV-positive patients in South Africa and Cameroon. Diagn Microbiol Infect Dis 79: 222-227, 2014.

9. Ribeiro RAL, de Alencar Menezes TO, de Melo Alves-Junior S, de Menezes SA, Marques-da-Silva SH and Rosário Vallinoto AC: Oral carriage of Candida species in HIV-infected patients during highly active antiretroviral therapy (HAART) in Belém, Brazil. Oral Surg Oral Med Oral Pathol Oral Radiol 120: 29-33, 2015.

10. Sun H, Chen Y, Zou X, Li H, Yin X, Qin H, Liu R, Yu C, Li Q, $\mathrm{Yu} \mathrm{K}$, et al: Occurrence of oral Candida colonization and its risk factors among patients with malignancies in China. Clin Oral Investig: Jul 30, 2015 (Epub ahead of print).
11. Tang J, Feng Y, Tsao S, Wang N, Curtain R and Wang Y: Berberine and Coptidis rhizoma as novel antineoplastic agents: A review of traditional use and biomedical investigations. J Ethnopharmacol 126: 5-17, 2009.

12. Liu R, Zhang X, Zhang L, Gao X, Li H, Shi J and Li X: Bitterness intensity prediction of berberine hydrochloride using an electronic tongue and a GA-BP neural network. Exp Ther Med 7: 1696-1702, 2014.

13. Kobayashi Y, Yamashita Y, Fujii N, Takaboshi K, Kawakami T, Kawamura M, Mizukami T and Nakano H: Inhibitors of DNA topoisomerase I and II isolated from the Coptis rhizomes. Planta Med 61: 414-418, 1995.

14. Iwasa K, Kamigauchi M, Ueki M and Taniguchi M: Antibacterial activity and structure-activity relationships of berberine analogs. Eur J Med Chem 31: 469-478, 1996.

15. Kim SH, Lee SJ, Lee JH, Sun WS and Kim JH: Antimicrobial activity of 9-O-acyl- and 9-O-alkylberberrubine derivatives. Planta Med 68: 277-281, 2002.

16. Park KD,Lee JH,Kim SH,Kang TH,Moon JS and Kim SU: Synthesis of 13-(substituted benzyl) berberine and berberrubine derivatives as antifungal agents. Bioorg Med Chem Lett 16: 3913-3916, 2006.

17. Gambari R, Hau DK, Wong WY and Chui CH: Sensitization of Hep3B hepatoma cells to cisplatin and doxorubicin by corilagin. Phytother Res 28: 781-783, 2014.

18. Darwish RM, Aburjai T, Al-Khalil S and Mahafzah A: Screening of antibiotic resistant inhibitors from local plant materials against two different strains of Staphylococcus aureus. J Ethnopharmacol 79: 359-364, 2002.

19. Yang ZC, Wang BC, Yang XS, Wang Q and Ran L: The synergistic activity of antibiotics combined with eight traditional Chinese medicines against two different strains of Staphylococcus aureus. Colloids Surf B Biointerfaces 41: 79-81, 2005.

20. Mandal S, DebMandal M, Pal NK and Saha K: DebMandal M, Pal NK and Saha K: Synergistic anti-Staphylococcus aureus activity of amoxicillin in combination with Emblica officinalis and Nymphae odorata extracts. Asian Pac J Trop Dis 3: 711-714, 2010.

21. Mandal S, Mandal MD and Pal NK: Enhancing chloramphenicol and trimethoprim in vitro activity by Ocimum sanctum Linn. (Lamiaceae) leaf extract against Salmonella enterica serovar Typhi. Asian Pac J Trop Med 5: 220-224, 2012.

22. Bodiwala HS, Sabde S, Mitra D, Bhutani KK and Singh IP: Synthesis of 9-substituted derivatives of berberine as anti-HIV agents. Eur J Med Chem 46: 1045-1049, 2011.

23. NCCLS. Reference Method for Broth Dilution Antifungal Susceptibility Testing of Yeasts; Approved Standard. 2nd edition. National Committee for Clinical Laboratory Standards, Wayne, PA, M27-A2, 2002.

24. Zhu J, Krom BP, Sanglard D, Intapa C, Dawson CC, Peters BM, Shirtliff ME and Jabra-Rizk MA: Farnesol-induced apoptosis in Candida albicans is mediated by Cdr1-p extrusion and depletion of intracellular glutathione. PLoS One 6: e28830, 2011.

25. Chung PY, Gambari R, Chen YX, Cheng CH, Bian ZX, Chan AS, Tang JC, Leung PH, Chui CH and Lam KH: Development of 8-benzyloxy-substituted quinoline ethers and evaluation of their antimicrobial activities. Med Chem Res 24: 1568-1577, 2015.

26. Lam PL, Gambari R, Kok SH, Lam KH, Tang JC, Bian ZX, Lee KK and Chui CH: Non-toxic agarose/gelatin-based microencapsulation system containing gallic acid for antifungal application. Int J Mol Med 35: 503-510, 2015.

27. McClellan KJ, Wiseman LR and Markham A: Terbinafine. An update of its use in superficial mycoses. Drugs 58: 179-202, 1999.

28. Dhamgaye S, Devaux F, Vandeputte P, Khandelwal NK, Sanglard D, Mukhopadhyay G and Prasad R: Molecular mechanisms of action of herbal antifungal alkaloid berberine, in Candida albicans. PLoS One 9: e104554, 2014.

29. Wei GX, Xu X and Wu CD: In vitro synergism between berberine and miconazole against planktonic and biofilm Candida cultures. Arch Oral Biol 56: 565-572, 2011.

30. Iwazaki RS, Endo EH, Ueda-Nakamura T, Nakamura CV, Garcia LB and Filho BP: In vitro antifungal activity of the berberine and its synergism with fluconazole. Antonie van Leeuwenhoek 97: 201-205, 2010.

31. Quan H, Cao YY, Xu Z, Zhao JX, Gao PH, Qin XF and Jiang YY: Potent in vitro synergism of fluconazole and berberine chloride against clinical isolates of Candida albicans resistant to fluconazole. Antimicrob Agents Chemother 50: 1096-1099, 2006.

32. Li DD, Xu Y, Zhang DZ, Quan H, Mylonakis E, Hu DD, Li MB, Zhao LX, Zhu LH, Wang Y, et al: Fluconazole assists berberine to kill fluconazole-resistant Candida albicans. Antimicrob Agents Chemother 57: 6016-6027, 2013. 